\title{
Reliability of an All-Optical Differential Current Detection Technique during Environmental Temperature Perturbations
}

\author{
Grzegorz Fusiek, Member, IEEE, Philip Orr and Paweł Niewczas Member, IEEE, \\ Institute for Energy and Environment, Department of Electronic and Electrical Engineering, \\ University of Strathclyde, Glasgow G1 1XW, United Kingdom \\ g.fusiek@strath.ac.uk
}

\begin{abstract}
The reliability of a novel, all-optical differential current protection scheme over varying environmental conditions is investigated in this paper. By monitoring the optical power reflected from two matched hybrid fiber Bragg grating current sensors and using a simple and low-cost optoelectronic threshold detector, a sub-cycle response to an increase in differential current can be achieved. A preliminary laboratory embodiment is constructed in order to emulate a practical deployment of the sensors and characterize the performance of the scheme when the sensors are at different temperatures. We demonstrate that even at a temperature difference of $20^{\circ} \mathrm{C}$ between the sensors the system is capable of robust and fastacting fault detection.
\end{abstract}

Keywords- fiber Bragg grating, piezoelectric transducer, voltage sensor, current sensor, optical, differential protection

\section{INTRODUCTION}

Differential unit protection is widely used in both terrestrial and marine power systems for transmission lines, transformers, tapped lines, motors, generators or busbars protection. It relies on the measurement and comparison of currents at two extreme locations of a protected zone. These currents will differ in magnitude, phase or both during fault occurrence [1].

A number of problems related to high-speed communication and sampling synchronization for microprocessor-based differential relays or the accuracy and speed of fault detection and tripping have been identified, and attempts have been made to improve the performance of existing protection systems by employing GPS (Global Positioning System) synchronization [2] and novel fault detection algorithms or fault detection schemes [3]-[7].

As an alternative to conventional protection systems, we have previously developed optical voltage and current sensors for different protection applications, including differential and distance protection schemes and demonstrated the potential for such optical techniques to overcome the complexities and bottlenecks associated with conventional relays' communication and synchronization requirements [8]-[10].

To reduce the cost of the aforementioned optical sensor systems by eliminating tunable devices for FBG centerwavelength tracking, we have recently proposed a novel alloptical fault detection scheme that can be used in applications where detailed information about each individual measured current is not required and, instead, detection of only the differential current is sufficient. Importantly, the speed of operation of an associated protection scheme could be extremely fast due to the all-optical nature of the differential calculation, and the simplicity of the optoelectronic or software threshold [11].

Preliminary laboratory tests of the proposed all-optical protection scheme carried out at room temperature demonstrated its high potential for fast-acting differential unit protection [11]. However, in a practical deployment, the sensors are likely to be at different temperatures due, for example, to their spatial separation within a protected zone. The FBG peak shifts due to temperature could, in this instance, introduce errors in fault detection and false tripping. Therefore, in this paper, we address these temperature-related issues and demonstrate that the proposed all-optical differential current detection can ensure reliable fault detection even when subject to varying environmental conditions.

\section{MEASUREMENT SYSTEM}

\section{A. Optical voltage and current sensors}

To monitor current at the ends of a protected zone, hybrid fiber-optic voltage and current sensors are employed [12], [13]. The optical voltage sensor utilizes a piezoelectric stack (transducer) and a fiber Bragg grating (FBG) sensor bonded to its surface as shown in Fig. 1(a). The strain generated by a voltage across the stack and exerted on the FBG produces a corresponding shift in its peak reflected wavelength. Thus, the peak wavelength shift can be calibrated in terms of voltage. By monitoring voltage across a burden resistor connected to the output of a small ferrite core current transformer (CT), as shown in Fig. 1(b), an optical current sensor can be realized whereby the FBG wavelength shift may be related to current.
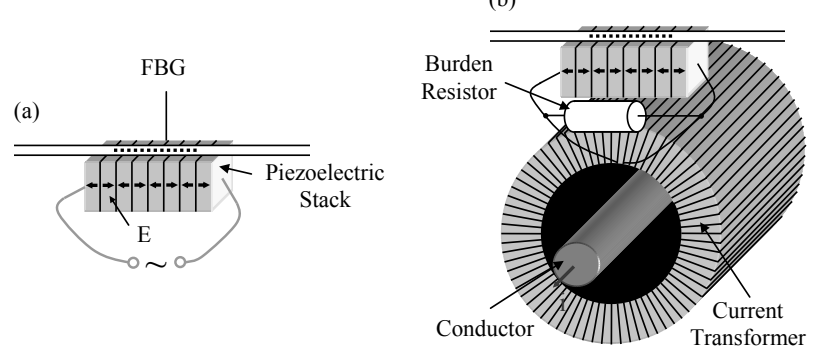

Fig. 1. Optical (a) voltage and (b) current sensors. 


\section{B. Optical fault detection concept}

The novel concept of all-optical fault detection relies on the use of a pair of matched current sensors utilizing two identical FBG sensors attached to piezoelectric transducers to monitor currents at the two extreme locations of the protected zone (see Fig. 2) [11].

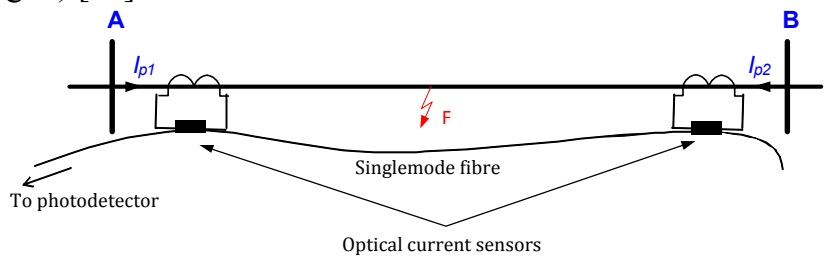

Fig.2. Fault detection scheme.

Under nominal (no fault) conditions, both current sensors detect identical ac currents and therefore the reflected sensor wavelengths shift identically - provided that the sensors are kept at the same temperature. The power recorded by the photodetector in this case is constant, since the integrated reflected power is constant while FBG reflections overlap as shown in Fig. 3(a). Under fault conditions, the sensors detect currents having either different amplitudes or different phases (or both), and hence the total reflected power is modulated depending on the separation of FBG spectra and the degree to which they overlap (Fig 3 (b)). This translates to a modulation of the photodetector output voltage which, after thresholding, may be used for fault indication. Thus, by using two identical current sensors placed at the boundaries of the protected zone, a differential current sensor is produced, which may be utilized as a fast-acting and lightweight all-optical differential fault detection scheme [11].

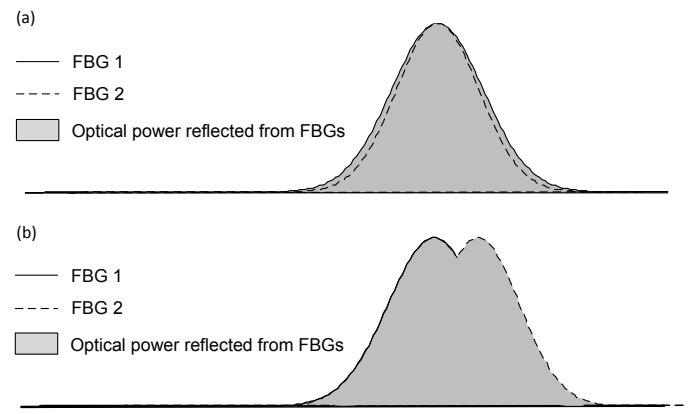

Fig. 3. Optical power distribution between two FBGs: (a) nominal condition FBGs are aligned, (b) fault condition - FBG reflections are separated.

\section{THERMAL EFFECTS}

To determine the hybrid sensor response to voltage and temperature a number of effects must be considered. A relative change in the FBG peak wavelength, $\Delta \lambda_{B} / \lambda_{B}$, due to a change in strain, $\varepsilon$, and temperature, $\Delta T$, can be expressed by $\Delta \lambda_{B} / \lambda_{B}=$ $C_{\varepsilon} \cdot \Delta \varepsilon+C_{T} \cdot \Delta T$, where $C_{\varepsilon}$ and $C_{T}$ are the strain and temperature sensitivities. The strain induced in the piezoelectric transducer by an external electric field under no mechanical stress can be expressed by $\varepsilon=d_{33} \cdot E=d_{33} \cdot V / l$, where $d_{33}$ is the longitudinal piezoelectric charge constant, $E$ is the electric field, $V$ is the voltage applied across the piezoelectric material and $l$ is the length of the material [12].
An FBG peak wavelength shift due to change in temperature around the hybrid voltage sensor stems from the thermal dependence of the FBG strain response, the thermal dependence of the piezoelectric charge constant, the thermal dependence of thermo-optic coefficient of fiber, and the thermal expansion of the piezoelectric and fiber materials [14][17]. As previously shown, the temperature response of the hybrid voltage sensor is predominantly governed by the thermo-optic coefficient and was estimated at around $10 \mathrm{pm} /{ }^{\circ} \mathrm{C}$ over the temperature range of $20^{\circ} \mathrm{C}$ to $100^{\circ} \mathrm{C}$ [17]. Consequently, this value was adopted in the simulations discussed in more detail in Section V.

\section{FAULT SCENARIOS}

We previously investigated a number of different fault scenarios, of which three representative cases have been summarized here and are discussed briefly below [11]. As shown in TABLE I. during the pre-fault condition both sensors were subjected to voltages of the same amplitude and phase. The fault condition was achieved by subjecting the sensors to voltages of different amplitude and/or phase to simulate the conditions of an internal fault. It should be noted that the voltages listed in TABLE I are matched to the voltage ratings of the piezoelectric transducers employed in the experimental investigation, such that the maximum possible sensitivity to a fault may be achieved. In practice, lower voltage transducers (e.g., $10 \mathrm{~V}$ rating) would likely be employed in conjunction with commercially-available CTs.

TABLE I. PRe-Fault and Fault Conditions

\begin{tabular}{l|c|c|c|c|c|c}
\hline \multirow{2}{*}{ Condition } & \multicolumn{3}{|c}{ Pre-Fault } & \multicolumn{3}{c}{ Fault } \\
& FBG1 & FBG2 & Phase & FBG1 & FBG2 & Phase \\
\hline Fault A & $200 \mathrm{~V}$ & $200 \mathrm{~V}$ & $0^{\circ}$ & $0 \mathrm{~V}$ & $300 \mathrm{~V}$ & $0^{\circ}$ \\
Fault B & $200 \mathrm{~V}$ & $200 \mathrm{~V}$ & $0^{\circ}$ & $200 \mathrm{~V}$ & $200 \mathrm{~V}$ & $180^{\circ}$ \\
Fault C & $200 \mathrm{~V}$ & $200 \mathrm{~V}$ & $0^{\circ}$ & $400 \mathrm{~V}$ & $300 \mathrm{~V}$ & $180^{\circ}$ \\
\hline
\end{tabular}

\section{SOFTWARE SIMULATION}

To theoretically evaluate the ability of the proposed alloptical fault detection method to withstand a temperature differential between the sensors, the equations from Section III were implemented in a dedicated LabVIEW simulation program [11]. The FBG spectral profiles were approximated by normalized Gaussian functions, and the light source illuminating the sensors was assumed to have a constant spectral profile [11]. The sensor sensitivity to temperature was assumed to be $10 \mathrm{pm} /{ }^{\circ} \mathrm{C}$, and its sensitivity to voltage was assumed to be $0.36 \mathrm{pm} / \mathrm{V}$ [12]. The normalized total optical power reflected from two FBG sensors having $0.2 \mathrm{~nm}$ bandwidths as a function of the spectral spacing between them is shown in Fig. 4 (solid line). The sensor working range is relatively narrow and the optical power modulation is not detectable when the spacing between the peaks is greater than $0.35 \mathrm{~nm}$. Such an FBG relative peak shift can be expected at a differential temperature change of $35^{\circ} \mathrm{C}$. By using FBGs with wider bandwidths, e.g. 1-nm bandwidths (dashed line in Fig. 4), the working range can be extended allowing for fault detection at a larger temperature difference between the sensors [11]. 


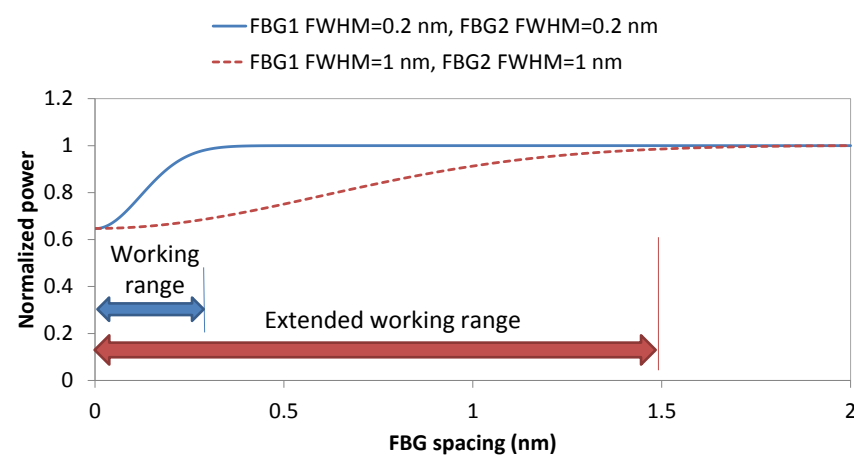

Fig. 4. Normalized optical power levels versus FBG spacing for two FBGs with different bandwidths. Wider FBG bandwidths extend fault detection working range.

An example of the simulated sensors' response to Fault C scenario (see Section IV) at a temperature difference of $20{ }^{\circ} \mathrm{C}$ between the sensors is shown in Fig. 5. This scenario represents a situation where power generation is present at each end of the faulted zone. In this case, fault inception results in the reversal of power flow at one end (towards the fault). By detecting the resulting change in the optical power modulation, the fault occurrence can be detected and the trip signal produced.

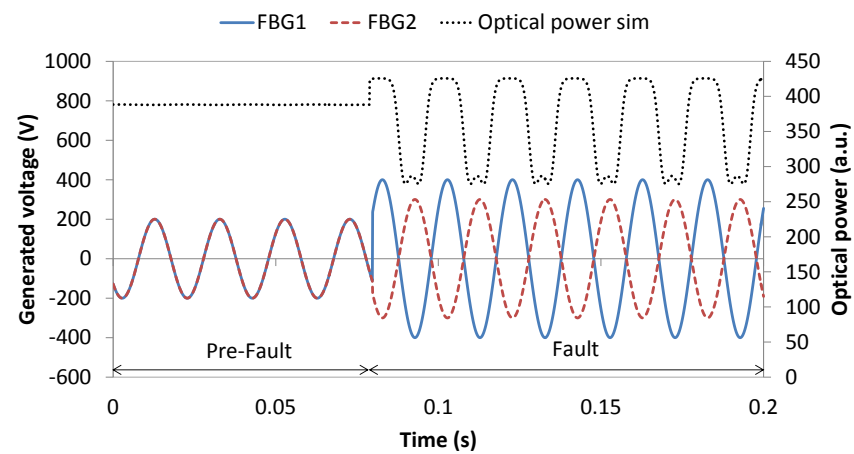

Fig. 5. Simulated sensors response to Fault $\mathrm{C}$ scenario for a temperature difference of $20^{\circ} \mathrm{C}$ between the sensors.

\section{EXPERIMENTAL RESULTS}

To demonstrate the reliability of the proposed fault detection technique for the sensors experiencing different environmental conditions, the experimental setup shown in Fig. 6 was constructed. Since current readings are achieved by measuring voltage across the burden resistor of the $\mathrm{CT}$, and the relation between voltage and current is linear, the electrical current-to-voltage conversion element was removed in the following tests to simplify the experimental circuitry [11].

Two FBG-based sensors were illuminated by a broadband super-fluorescent source having central wavelength at $1550 \mathrm{~nm}$ and $40 \mathrm{~nm}$ bandwidth. One of the sensors was kept at room temperature $\left(25^{\circ} \mathrm{C}\right)$, while the other was placed in an oven and kept at $45^{\circ} \mathrm{C}$. The optical signals reflected from the sensors - having bandwidths of $0.2 \mathrm{~nm}$ and spectral peaks at $1549.69 \mathrm{~nm}$ and $1549.74 \mathrm{~nm}$ - were monitored and converted to the electrical signal by means of a single photodetector. A 16-bit PXI data acquisition card and a PXI controller (both from National Instruments) were used to acquire signals from the photodetector which were then processed in a PC. The data acquisition sampling rate was set at $10 \mathrm{kS} / \mathrm{s}$. An amplifier module (APTS3AI, Relay Engineering Services Ltd) was used to generate voltage waveforms modeled in the $\mathrm{PC}$ and generated by the PXI unit.

The results of three different fault scenarios discussed in Section IV at a temperature difference of $20^{\circ} \mathrm{C}$ between the sensors are shown in Fig. 7.

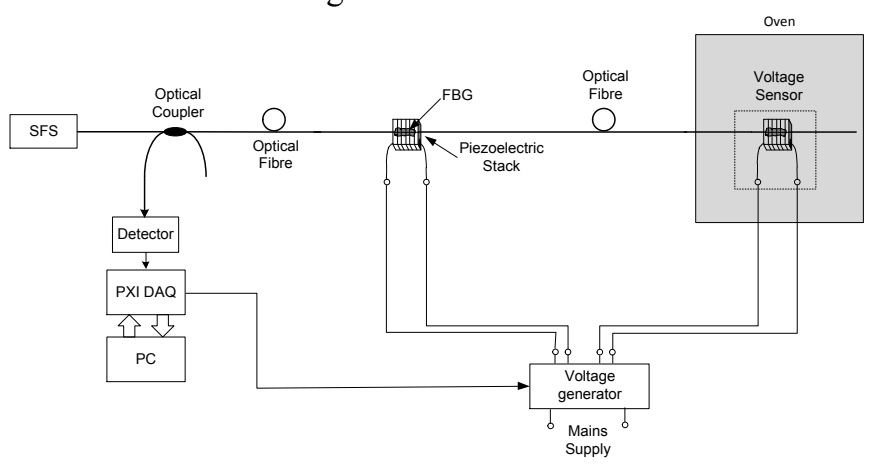

Fig. 6. Experimental setup
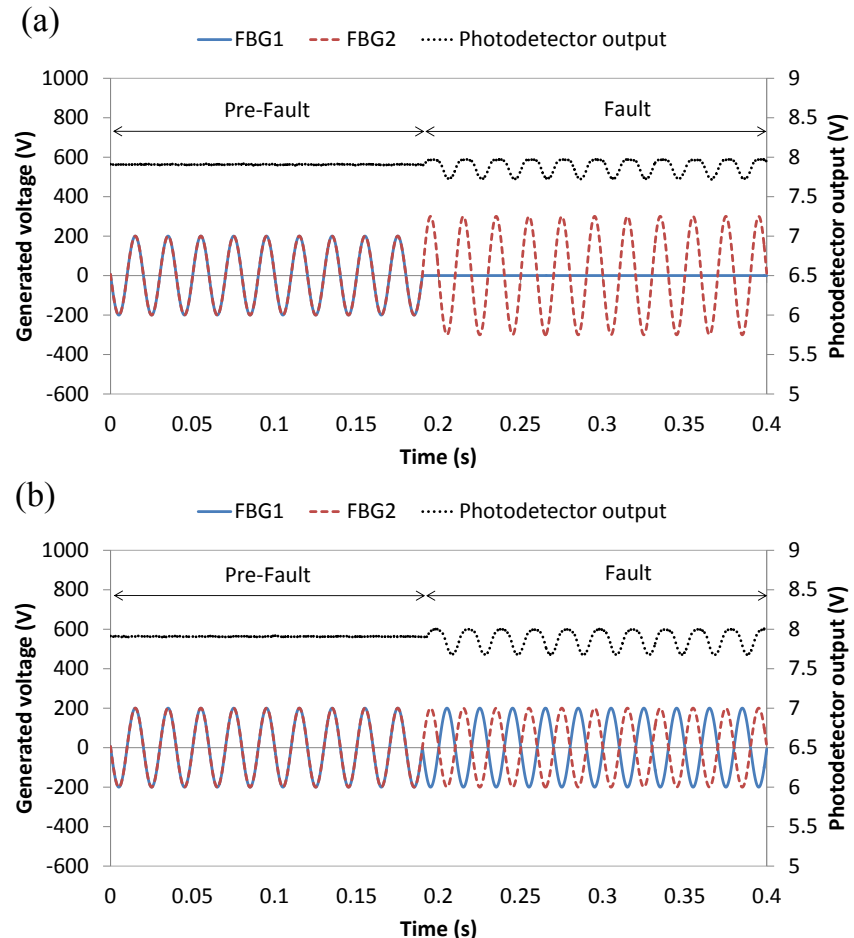

(c)

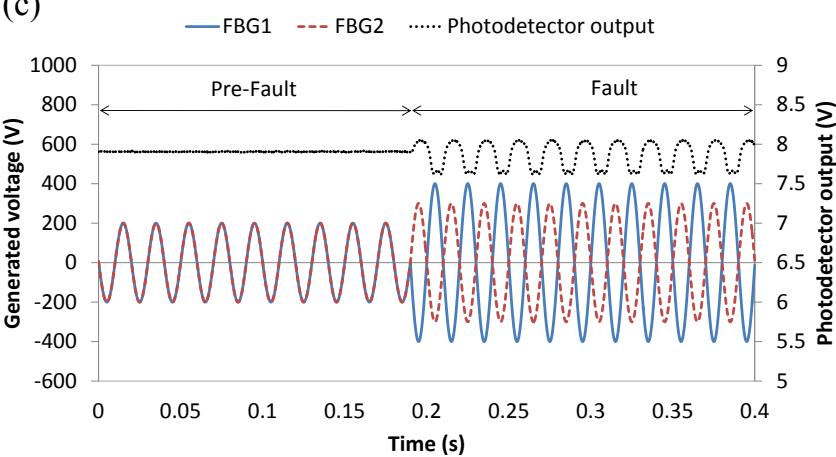

Fig. 7. Photodetector output (top trace) and voltage on the sensors (bottom traces) during (a) Fault A, (b) Fault B and (c) Fault C scenarios. The temperature difference between sensors is $20^{\circ} \mathrm{C}$. 
In the figure, the bottom traces represent $50-\mathrm{Hz}$ voltage waveforms modeled in the PC and captured immediately prior to generation by the PXI unit. These signals are then amplified by the APTS unit before they can be measured by the optical sensors. To generate the trip signal (Fig. 8), the photodetector output modulation was monitored by tracing peak-to-peak values of the signal and comparing these to a set threshold. Peak-to-peak values were estimated from the data length equal to one period of the voltage waveform, buffered and updated every $100 \mu$ s so that the trip signal could be generated with $100 \mu$ s delay after the threshold condition was met. The threshold level is adjustable and can be set according to the difference between peak-to-peak values of the acquired photodetector output in pre-fault and fault conditions. The experimental results are in good agreement with the simulations as shown in Fig. 9.

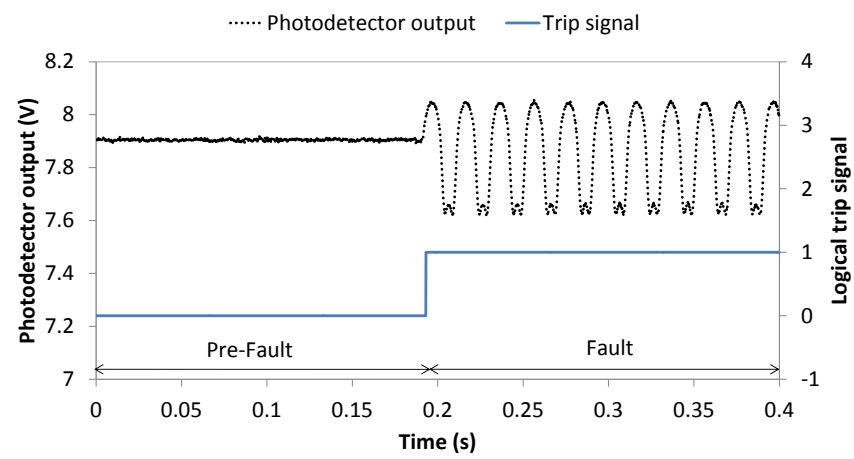

Fig. 8.Trip signal generation in response to Fault $\mathrm{C}$ detection. The temperature difference between sensors is $20^{\circ} \mathrm{C}$.

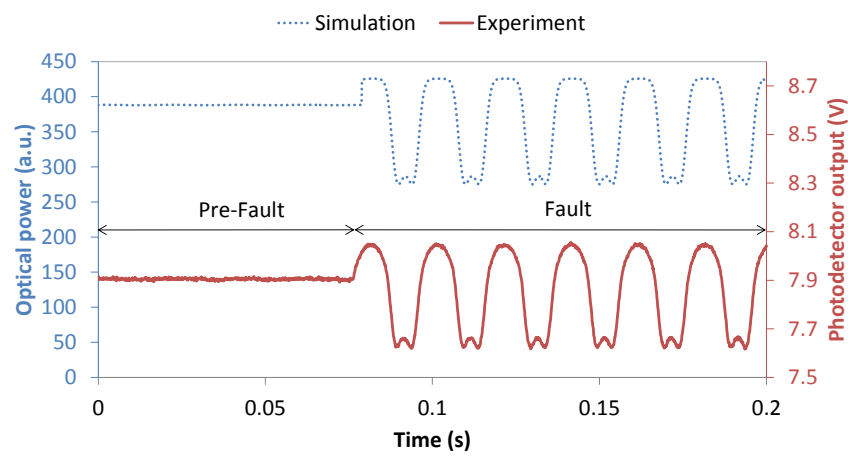

Fig. 9. Comparison between simulated (top trace) and experimental (bottom trace) photodetector outputs in response to Fault $\mathrm{C}$ scenario for a temperature difference of $20^{\circ} \mathrm{C}$ between sensors.

\section{CONCLUSIONS}

In this paper, we have theoretically and experimentally investigated the robustness of a novel all-optical differential unit protection method employing a distributed optical current sensing scheme. The practical deployment of this scheme was simulated in the laboratory by placing sensors at different temperatures which may result in practice from ambient temperature differentials over the length of an outdoor electrical circuit. By monitoring the reflected optical power modulation and comparing it to a set threshold, fast fault detection has been demonstrated even when the sensors experienced a temperature difference of $20^{\circ} \mathrm{C}$. Three different fault scenarios evaluated in the laboratory have shown that the new technique has the potential to enable fast-acting and inexpensive unit protection for electrical networks that operates reliably even under varying or unpredictable environmental conditions.

\section{REFERENCES}

[1] AREVA T\&D, Network Protection and Automation Guide. $1^{\text {st }}$ Ed., Cayfosa, Barcelona, ISBN 2-9518589-0-6, July 2002

[2] I. Hall, P. G. Beaumont, G. P. Baber, I. Shuto, M. Saga, K. Okuno, H. Ito, "New Line Current Differential Relay using GPS Synchronization”, 2003 IEEE Bologna Power Tech Conference,

[3] C.D. Booth; I. Elders; A. Mackay; J.D. Schuddebeurs; J.R. McDonald, "Power System Protection of All Electric Marine Systems," DPSP 2008

[4] Liu HanYu; Mu LongHua, "Integrated protection on shipboard integrated power system," APAP 2011

[5] L. Kumpulainen; T. Harju; H. Pursch; S. Wolfram, "High speed protection concept to minimize the impacts of arc-flash incidents in electrical systems of ships," ESTS 2011

[6] B. Kasztenny; K. Kuras, "A new algorithm for digital lowimpedance protection of busbars," Power Engineering Society Summer Meeting, 2001 , (1), 97-102, (2001)

[7] M.S. Sachdev; T.S. Sidhu; H.S. Gill, "A busbar protection technique and its performance during $\mathrm{CT}$ saturation and $\mathrm{CT}$ ratio-mismatch," Power Delivery, IEEE Transactions on , 15(3), 895-901, ( 2000)

[8] P. Orr, P. Niewczas, A. Dysko, C. Booth, "FBG-based fiberoptic current sensors for power systems protection: Laboratory evaluation," UPEC 2009

[9] P. Orr, G. Fusiek, P. Niewczas, A. Dysko, C. Booth, F. Kawano, G. Baber, "Distributed optical distance protection using FBGbased voltage and current transducers," PES GM 2011

[10] P. Orr, G. Fusiek, C.D. Booth, P. Niewczas, A. Dyśko, F. Kawano, P. Beaumont, T. Nishida, 'Flexible protection architectures using distributed optical sensors', IET Conf. Pub. 2012, 155 (2012)

[11] G. Fusiek, P. Orr, H. Wang, P. Niewczas, "All-optical Differential Current Detection Technique for Unit Protection Applications", I2MTC 2013

[12] L. Dziuda, P. Niewczas, G. Fusiek, J. R. McDonald, "Hybrid Fiber-Optic Voltage Sensor for Remote Monitoring of Electrical submersible Pump Motors”, Optical Engineering, 44(6), (2005)

[13] L. Dziuda, G. Fusiek, P. Niewczas, G. Burt, and J.R. McDonald, "Laboratory Evaluation of the Hybrid Fiber-Optic Current Sensor", Sensors and Actuators, A: Physical, 136(1), (2007)

[14] M. J. O’Dwyer, C. Ye, S. W. James, R. P. Tatam, "Thermal dependence of strain response of optical fibre Bragg gratings", Measurement Science and Technology, 15, (2004)

[15] X. Dong, Y. Liu, Z. Liu, and X. Dong, "Simultaneous displacement and temperature measurement with cantileverbased Fiber Bragg grating sensor," Opt. Comms, 192, (2001).

[16] S. Magne, S. Rougeault, M. Vilela, and P. Ferdinand, "State-ofstrain evaluation with fiber Bragg grating rosettes: application to discrimination between strain and temperature effects in fiber sensors," Applied Optics, 36(36), (1997).

[17] Niewczas, L. Dziuda, G. Fusiek, J.R. McDonald, "Temperature Compensation for a Piezoelectric Fiber-Optic Voltage Sensor," IMTC 2006 